yan.tex

\title{
Numerical observation of non-axisymmetric vesicles in fluid membranes
}

\author{
Yan Jie ${ }^{1}$, Liu Quanhui ${ }^{1}$, Liu Jixing ${ }^{1}$, Ou-Yang Zhong-can ${ }^{1,2}$ \\ ${ }^{1}$ Institute of Theoretical Physics, Chinese Academy of Sciences, \\ P.O. Box 2735, Beijing 100080, China \\ ${ }^{2}$ Center for Advanced Study, Tsinghua University, Beijing 100084, China
}

(August 9, 2018)

\begin{abstract}
By means of Surface Evolver (Exp. Math,1,141 1992), a software package of bruteforce energy minimization over a triangulated surface developed by the geometry center of University of Minnesota, we have numerically searched the non-axisymmetric shapes under the Helfrich spontaneous curvature (SC) energy model. We show for the first time there are abundant mechanically stable non-axisymmetric vesicles in SC model, including regular ones with intrinsic geometric symmetry and complex irregular ones. We report in this paper several interesting shapes including a corniculate shape with six corns, a quadri-concave shape, a shape resembling sickle cells, and a shape resembling acanthocytes. As far as we know, these shapes have not been theoretically obtained by any curvature model before. In addition, the role of the spontaneous curvature in the formation of irregular crenated vesicles has been studied. The results shows a positive spontaneous curvature may be a necessary condition to keep an irregular crenated shape being mechanically stable.
\end{abstract}

PACS numbers: 87. 22. Bt, 62. 20. Dc, 02. 60. -x 


\section{INTRODUCTION}

Vesicles are bags of lipid bilayer membranes which form spontaneous in aqueous environment under appropriate conditions. In order to study theoretically the morphology of vesicles, a lipid bilayer which has liquid crystalline structures and characteristics have long been considered as a model. The first description of fluid membrane by a curvature energy model was given by Canham[1], in which the local energy density of the form $(2 H)^{2}$ was introduced, where $H$ is the mean curvature of the surface. From the current prospective, this energy model is a faithful description of a vesicle which consists of a symmetric bilayer. However, real lipid bilayers are not symmetric and thus there is not a genuine physical realization of this model. Helfrich [2] proposed from curvature elastic theory in liquid crystal the well-known spontaneous curvature (SC) energy model, in which the energy functional is

$$
F=\frac{1}{2} \kappa_{c} \int\left(C_{1}+C_{2}-C_{0}\right)^{2} d A+\Delta P \int d V+\lambda \int d A,
$$

where $d A$ and $d V$ are the surface area and the volume elements, respectively, $\kappa_{c}$ is an elastic modulus, $C_{1}$ and $C_{2}$ are the two principal curvatures, and $C_{0}$ is the spontaneous curvature which describes the possible asymmetry of the bilayer membrane. Nonzero values of the spontaneous curvature results from the fact that a lipid bilayer may have a tendency to curve one way or the other, due, for example, either to intrinsic chemical asymmetry between the two leaves and/or to a chemical asymmetry between the interior and exterior aqueous environments. The Lagrange multipliers $\Delta P$ and $\lambda$ take account of the constraints of constant volume and constant area, which can be physically understood as the osmotic pressure between the ambient and the internal environment, and the tensile coefficient, respectively. Based on the model, many works have been done in the axisymmetric case. In their pioneering work on the model, Deuling and Helfrich [3-4] numerically found a catalog of possible axisymmetric vesicles shapes. In their papers they used the nomenclature developed in red blood cells to describe the shapes they found. Among these shapes were prolate and oblate ellipsoid, stomatocytes, and discocytes, some of which resemble the shapes of human red blood cells (RBCs). 
Locating different branches of shapes of minimal energy in the parameter space, then the division of the parameter space represents the so-called phase diagram. In SC model, Seifert et al [5] calculated the two-dimensional phase diagram for axisymmetric shapes in SC model within a limited parameter space. The parameters they used are the reduced volume $v$ and the scaled spontaneous curvature $c_{0}$, which are defined as $v=\frac{V}{(4 / 3) \pi R_{0}^{3}}, c_{0}=C_{0} R_{0}$, where $R_{0}$ is defined by $A=4 \pi R_{0}^{2}$ and $A, V$ are the real volume and area of the surface, respectively.

In addition, by performing the variation of the energy functional the general equilibrium shape equation was derived [6]

$$
\Delta p+2 \lambda H-2 k_{c}\left[2 H\left(H^{2}-K\right)+C_{0} K+\left(C_{0}^{2} / 2\right) H+\Delta H\right]=0,
$$

where the operator $\Delta$ is the Laplace-Beltrami operator $\Delta=\left(\frac{1}{\sqrt{g}} \partial_{i}\left(g^{i j} \sqrt{g} \partial_{j}\right), g\right.$ is the determinant of the metric $g_{i j}$ and $g^{i j}=\left(g_{i j}\right)^{-1}, K=C_{1} C_{2}$ is the Gaussian curvature and $H=(1 / 2)\left(c_{1}+c_{2}\right)$ is the mean curvature (Here we use a different sign convention for $H$ from the original derivation of this general shape equation in [6]).

Solving the shape equation under the axisymmetric case (the corresponding shape equation can be transformed from (2) into an ordinary differential equation [7]), several analytical solutions have been found. Among these solutions are the solution of biconcave shape, the torus solution, and the beyond Delaunay surfaces [8]. The first two solution have been supported experimentally [9] and the third is believed to be a hunt for experiments. All these studies concentrate on axisymmetric vesicle shapes. No axi-symmetric vesicle shapes of spherical topology has been reported in SC model.

On the other hand, in other curvature energy models, some progress has been achieved in finding non-axisymmetric vesicle shapes of spherical topology by means of brute-force energy minimization over a triangulated surface. In area difference elastic (ADE) model, non-axisymmetric ellipsoid shapes have been reported [10]. very recently, in a modified ADE model including the contribution of the compressibility of the total area and volume, Wintz et al [11] reported a catalog of starfish shapes. Characteristic for such shapes is their flatness and their multi-fold symmetry. As far as we know, no other non-axisymmetric vesicle shapes 
have been reported in literature by any curvature model.

However, on the experimental side, abundent non-axisymmetric RBC shapes of spherical topology have been observed. They may take very complex shapes and many of them even have no any intrinsic geometric symmetry. There are a lot of clear images obtained by scanning electron microscope of RBCs in the book "Living Blood Cells and their Ultrastructure" [12] including very complex vesicle shapes such as echinocyte type cells (Fig.98 in [12]) which have a characteristic shape with crenations or spicules (nearly) evenly distributed on the surface, acanthocyte type cells (Fig. 157, Fig. 159 in [12]) which bear a superficial resemblance to echinocytes but with much fewer spicules irregularly arranged and bent back at their tips, Knizocytes (Fig 106, Fig. 107 in [12]) which are tri- and quadri-concave shapes, and Sickle type cells (Fig.198 in [12]) which show a sickle-like shape, et al. These complex shapes are not yet understood theoretically in the context of bending energy models. Some researchers [13] guess that such exotic shapes maybe involve other energy contributions such as higher-order-curvature terms and van der Waals attraction of the membrane. However, the conjecture is not so obvious as it seems. Our question is: is it possible that these complex shapes can be described by a simple curvature model, e.g., SC model? It is the purpose of this paper to numerically search non-axisymmetric shapes of spherical topology in SC model.

Because of its success in finding non-axisymmetric ellipsoidal shape and starfish vesicle, we will also employed the algorithm of brute-force energy minimization over a triangulated surface in this study. Such a method directly minimizes the total energy. The resulting shape is a local energy minimum, which depends in principle on the initial shape chosen.

We will also locate each shapes found by us in the phase diagram in order to know about in which region of the parameter space these shapes exist. In addition, in order to describe vesicle shapes, we will use the same nomenclature as in [12].

The plan of the paper is: Section II describes the algorithm and the procedure; Section III gives the main results; Section IV is the discussion and conclusion. 


\section{THE MODEL, THE SOFTWARE AND THE PROCEDURE}

In order to find the the locally stable non-axisymmetric configurations of RBCs, we evaluate the bending energy numerically with constraint of the constant volume or constant area based on the SC model. The reason that we don't require the constant volume and constant area simultaneously is to avoid possible contradictions of the two constraints in our procedure described below. Under the constraint of constant volume $V$, the parameter $\lambda$ is just be understood the tensile coefficient, and, under the constraint of constant area $A$, the parameter $\Delta P$ is understood as the osmotic pressure. Since our main purpose is to search possible non-axisymmetric shapes in SC model instead of to study shape transitions, such a choice is applicable. To our experience, by our procedure described below the software we used in this study performances better at the constraint of constant volume than at that of constant area, so in most cases we adopt the constraint of constant volume.

The software we used to searching the surfaces is "Surface Evolver" package of computer programs [14], which is based on a discretization of the curvature energy, area, and volume on a triangulated surface. The energy in the "Evolver" can be a combination of surface tension, gravitational energy, squared mean curvature et al. Constraints can be geometrical constraints on vertex positions or constraints on integrated quantities such as body volumes, surface area, et al. The constraints are incorporated into the bending energy and the corresponding Lagrange multipliers will be reported. The resulting total energy is minimized by a gradient descent procedure. The resulting shape is a local energy minimum. These characteristics of the "Evolver" make it be an useful tool for for studying non-axisymmetric shapes in SC model. In the "Evolver" the osmotic pressure is denoted by an internal pressure $P$, and it can deal with the following energy functional conveniently

$$
F=m_{1} \int\left(H-H_{0}\right)^{2} d A+\lambda \int d A-P \int d V
$$

where $m_{1}$ is called the "weight" of the bending energy. Under the definition of $H=$ $(1 / 2)\left(C_{1}+C_{2}\right)$, The model is identical to SC model by the transformations: $m_{1}=2 \kappa_{c}$, 
$P=-\Delta P$, and $H_{0}=C_{0} / 2$. In addition, no particular units of measurement are used in the "Evolver". In order to relate the program values to the real world, then all values should be within one consistent unit system.

The software has been employed to deal many geometry problems such as constant mean curvature surfaces, equilibrium foam structure at al. for several years [15-17], so we can rely on it safely. Just as an exercise, we have tested it for the equilibrium condition of a perfect sphere with a given target volume evolved from a cube in the SC model. The equilibrium condition for the energy functional (3) is

$$
-P r^{3}+2 \lambda r^{2}+2 m_{1} H_{0} r\left(-1+H_{0} r\right)=0,
$$

where $r$ is the radius of the sphere, which is identical to the results in [6]. Under the parameters $m_{1}=1, H_{0}=1, \lambda=2$, and the target volume $V=4.189$, we obtained a stable unit sphere with the area $A=12.5774$, and the lagrange multiplier $P=4.0023$, which is obviously satisfied the equilibrium condition.

On the other hand, the resulting shape generated by such an algorithm depends in principle on the initial shape one chooses. The dependence on the initial shape leads to a real difficulty to choose appropriate initial shape for yielding interesting configurations. In order to find new kinds of non-axisymmetric shapes, the strength of the dependence on the initial shape chosen must be reduced by some way. We adopt a procedure based on making the target configuration geometrically "far away" from an initial shape. Taking the constant volume constraint as an example,the detailed procedure is illustrated in the following:

i). Under certain values of $k_{c}, C_{0}, \lambda$, get (for example) a stable sphere with a given target value of the volume $V_{0}$.

ii). Taking the sphere as an initial shape, change the target volume to a value $V_{1}$ which is far from $V_{0}$. This is in order to break the stability of the sphere and make the target shape not being "adjacent" to the sphere. It is hoped that such a sudden and big change in the volume will trigger a "random walk" of the surface before it finally stops at a locally stable configuration with the volume $V_{1}$. 
iii). Each new generated shapes and the complex intermediate unstable shapes can also be taken as initial shape, so the procedure can leads to many shapes with no "correlation" among each other. Such a way is capable of finding many different kinds of surfaces from a simple initial shape.

The above procedure have generated many striking shapes, as reported in the next section.

\section{THE MAIN RESULTS}

By the procedure described in the last section, many striking vesicle shapes were found. Some of shapes qualititavily resemble the RBC shapes observed experimentally. In order to describe these exotic shapes, we also adopt the nomenclature devoloped in red blood cells. We want to report four types of shape in this paper which are corniculate shape, knizocyte type shape, sickle type shape, and acanthocyte type shape. We drew our intention to the last kind of shape, because it is strikingly irregular without any geometric symmetry and because it shows other interesting features such as "budding" and "vesiculation" formation. The role of the spontaneous curvature in the formation of acanthocyte type shapes is studied, which shows a positive spontaneous curvature may be a necessary condition for the formation of such exotic shapes. Each shape is mapped into the two dimensional phase diagram (Fig.8) spanned by the reduced volume and the scaled spontaneous curvature.

\section{Corniculate shape}

Figure 1 shows a corniculate surface with six corns, whose location in the phase diagram is $\left(v=0.93, c_{0}=1.28\right)$ denoted by "* 1 " in Fig.8. We obtain it from a destabilized sphere induced by osmotic pressure, without the constraints of constant area and constant volume. The corresponding paprameters are $\left(P=-4, \lambda=0.5, H_{0}=1, m_{1}=0.5, A=5.15, V=\right.$ 1.02). This shape apparently has rotational symmetry which is identical to the octahedron and thus isomorphic to $S_{4}$, where $S_{X}$ denote the group of permutations of the set $X$ [18]. 
Though we didn't find the shape in [12], we hope it will be a hunt for future experiments. In addition, such a shape maybe indicate the way of formation of echinocyte III vesicle shapes (Fig.98-Fig.100 in [12]) which have 10-50 corns evenly distributed on a nearly spherical surface.

\section{Knizocyte type shape}

Knizocytes (Fig 106, Fig. 107 in [12]) are tri- and quadri-concave shapes found in the experiments of RBCs. The shapes denoted by Figure 2 is a quadri-concave shapes and bears a resemblance of the experimentally observed shape (Fin.106 in [12]). Its location in the phase diagram is $\left(v=0.84, c_{0}=-1.41\right)$ denoted by "*2" in Fig.8. We obtained it by under the constraint of constant volume from an initial shape of a smaller volume. The corresponding paprameters are $\left(P=4.09, \lambda=2, H_{0}=-0.5, m_{1}=1, A=25.10, V=10\right)$. The shape has rotational symmetry which is identical to the cube and also isomorphic to $S_{4}[18]$. Such a shape may be seen under different circumstances. In fresh blood, it may be observed in certain hemolytic anemias. In addition, if a suspension of cells is examined between slide and coverslip and an erythrocyte permitted to adhere to the slide, gentle deformation of the cell by a current of liquid in the preparation may produce this appearance [19].

\section{Sickle type shape}

Figure 3 bears a resemblance of the sickle cells in echinocytic forms (Fig.198 in [12]). The location in the phase diagram is $\left(v=0.74, c_{0}=-1.48\right)$ and denoted by "**" in Fig.8. It is obtained under the constraint of constant volume from an initial shape with much smaller volume. The corresponding paprameters are $\left(P=5.03, \lambda=1.75, H_{0}=-0.5, m_{1}=1\right.$, $A=27.50, V=10)$. Sickle cell is related to sickle cell disease, a hereditary abnormality. Sickle cells appear when affected blood is exposed to a sufficiently low oxygen tension. The phenomenon can also be seen by sealing a preparation between slide and coverslip and waiting a few hours or leaving the blood for 24 to 48 hours in a vessel without oxygen [20]. 
Figure 4 shows a strikingly complex shape without any intrinsic geometric symmetry. Characteristic for this shape is its irregular characteristic shape and the several irregular distributed crenations, which are just the same as the so-called acanthocyte type cell shapes (Fig. 157, Fig. 159 in [12]) observed experimentally. The location of this shape in Fig.8 is $\left(v=0.56, c_{0}=1.89\right)$ denoted by "* 4 ". It is obtained by applying the procedure described in section II several times from a simpler initial shape under the constraint of constant volume of 2 . The corresponding parameters are $\left(P=8.21, \lambda=2.25, H_{0}=1, m_{1}=1\right.$, $A=11.24, V=2)$. The designation acanthocyte was given by Singer et al.[21] to crenated red cells found in an hereditary illness now characterized by the absence of beta-lipo-protein and serious nervous system alterations. The abnormality appears to develop during the lifespan of the cells within the circulation and to be absent or minimal in the youngest cells [22]. Another interesting point is that this shape shows clear "budding" and "vesiculation" formation, where the term "vesculation" has the same definition in [23], which distinguishes the (singular) limit at which the radius of the neck connecting the mother and the daughter vesicles becomes microscopic. Such abnormal shapes were found abundant in this study. In addition, the formation of such irregular shapes and "budding" as well as "vesiculation" seems closely related to the spontaneous curvature. We show this by the following several shapes evolved from figure 4 by changing the value of the spontaneous curvature. Figure 5 is the result by increasing the value of $H_{0}$ to $H_{0}=8$, from which one can see that all the buds and the crenations are evolved into "vesiculations". The location of this figure in Fig.8 is $\left(v=0.70, c_{0}=14.1\right)$ which is beyond the region of the phase diagram. The corresponding paprameters are $\left(P=182.00, \lambda=2.25, H_{0}=8, m_{1}=1, A=9.76, V=2\right)$. Figure 6 is the result evolved from figure 4 by setting $H_{0}=0$, which is a perfect sphere with $\left(P=5.75, \lambda=2.25, H_{0}=0, m_{1}=1, A=7.68, V=2\right)$. The location in Fig.8 is $(v=1.00$, $\left.c_{0}=0\right)$ denoted by "*6", which is a perfect sphere. One can also check the parameters by the equilibrium condition of a sphere in SC model. Figure 7 is the result evolved from figure 4 by setting $H_{0}=-2$, which is a pear like shape with $\left(P=24.88, \lambda=2.25, H_{0}=-2\right.$, $\left.m_{1}=1, A=8.31, V=2\right)$. The location in Fig.8 is $\left(v=0.89, c_{0}=-3.25\right)$ denoted by “* 7 ". 


\section{DISCUSSION AND CONCLUSIONS}

The algorithm used in this study has powerful ability to find complex vesicle shapes, providing that the dependence in the initial shape chosen can be broken by some way. Though our procedure used in the study can not completely break the dependence in the initial shape, many strikingly complex shapes in SC model have been found. Some shapes we searched bear the resemblance of experimentally observed RBC shapes. Apparently the procedure is also useful in searching new shapes of high genus and can be used in other curvature models. Though the shapes provided in this paper are all new, We have the most strong impression of the existence of irregular acanthocyte type in SC model, because this is the first irregular shapes described by a simple bending energy model. Our study shows that adding new energy contributions, such as higher-order-curvature terms and van der Waals attraction of the membrane, is not necessary to account for such anomous shapes. In fact, taking Figure 4 as the initial shape, we have obtained several other irregular shapes which are not included in this paper.

The reason why these shapes have not been reported by other researchers also employing the same algorithm is simple: by gradually changing the control parameters such as the reduced volume, the spontaneous curvature, et al. from a (simple/regular) initial shape, the resulting set of shapes obtained are all strongly correlated to each other, i.e., all of them belong to a "bifurcation tree" in the phase diagram. However, there may have coexisting stable configurations of different symmetry corresponding to a set of control parameters, each of them also leads to its own "bifurcation tree" in the phase diagram. Thus, a "jump" between these "trees" is required to find complex shapes.

We are very interested in the role of the spontaneous curvature in the formation of irregular crenated shapes like acanthocytes observed in RBC experiments (e.g., Fig.159, Fig.157, Fig.110 in [12]). The crenations of the acanthocytes in fact can be considered as the indication of budding formation. In literature, the theoretical study of budding transition has focused on the axisymmetric case. Under the axisymmetric condition, the physical 
origin of the budding transition from a sphere in SC model is easy to be understood. As area increases (or, as volume decreases) an excess area (compared with the area of a sphere with the same volume) is available. If this excess area becomes comparable to $4 \pi\left(2 / C_{0}\right)^{2}$, it becomes favorable to shed the excess area in the form of a bud with radius compared to $\left(2 / C_{0}\right.$, which costs very little energy [24]. According to the natural explanation of budding transition, a positive spontaneous curvature should be a necessary condition. Though in our case the shapes are not axisymmetric, the basic mechanism of shedding area into buds should be the same. In addition, since heavily distorted shapes correspond to large excess area, it is naturally to think that the mechanism of shedding area also accounts for the formation of such a heavily distorted shape. Because of the above reasons, we guess that a positive spontaneous curvature is also a necessary condition for the formation of acanthocytes. The results shown by Figure 5, figure 6, and figure 7 support this conjecture. In addition, we have tested the conjecture for several other irregular crenated shapes which are not included in this paper, all the results also support the conjecture. From the locations of these shapes in Fig.8, our shapes are mainly in the part of higher reduced volume. Fig.4 has the lowest value of $v$ among our shapes, which indicates that the most likely region of irregular shapes

is in lower part of $v$. It is equivalent to say that a large excess area $A\left(1-v^{\left(\frac{2}{3}\right)}\right.$ favours the formation of heavily distorted shapes, which is appreciable.

\section{ACKNOWLEDGMENTS}

We are indebted to Professor Ken Brakke, Professor Karsten Grosse-Brauckmann and Professor Rob Kusner for their guidance on the software and useful suggestions. We thank Dr. Zhou Haijun, Dr. Zhao Wei for fruitful discussions. This work is partly supported by the National Natural Science Foundation of China. 


\section{REFERENCES}

[1] Canham,P.B. J.Theor. Biol. 26, 61-81 (1970).

[2] W. Helfrich, Z. Naturforsch. 28C, 693 (1973).

[3] H.J. Deuling and W. Helfrich, Biophysics J., 16, 861 (1976).

[4] H.J. Deuling and W. Helfrich, J. De Phys. ., 37, 1335 (1976).

[5] U.Serfeit ,K. Berndl, and Lipowsky, Phys. Rev. A 44, 1182 (1991).

[6] Ou-Yang Zhong-Can and W.Helfrich, Phys. Rev.Lett.59, 2486 (1987); Phys. Rev. A 39, 5280 (1989).

[7] Hu Jian-Guo and Ou-Yang Zhong-Can, Phys. Rev. E 47, 461 (1993).

[8] H.Naito, M.Okuda, and Ou-Yang Zhong-Can, Phys. Rev. E 48, 2304 (1993);Ou-Yang Zhong-Can, Phys. Rev. A 41, 4517 (1990); H.Naito, M.Okuda, and Ou-Yang ZhongCan, Phys. Rev. Lett. 74, 4345 (1995).

[9] M. Mutz and D.Bensimon, Phys. Rev. A 43, 4525 (1991); A.S.Rudolph et al, Nature 352, 52 (1991); Z.Lin et al, Langmuir 10. 1008 (1994);

[10] Jaric, M. et al, Phys. Rev. E 52, 6623 (1995).

[11] Wintz et al, EuroPhys. Lett. 23, 404-408 (1996).

[12] M.Bessis, Living Blood Cells and their Ultrastructure, (Springer-Verlag, Berlin, 1973).

[13] Ling Miao, Udo Seifert, Michael Wortis, and Hans-Gunther Dobereiner, Phys. Rev. E., 49,5389 (1994).

[14] Brakke, K.A., Exp. Math,1,141 (1992).

[15] Lucas Hsu, Rob Kusner, John Sullivan, Experimental Math., 1, Issue 3 (1992).

[16] Karsten Grosse-Brauckmann, Konrad Pothier, Experimental Math., 6, Issue 1 (1997). 
[17] Robert Phelan, Denis Weaire, Kenneth Brakke, Experimental Math., 4, Issue 3 (1997).

[18] M. A. Armstrong, Groups and Symmetry, (Springer-Verlag, Berlin, 1988).

[19] Bull, B. S., Nouv. Rev. fr. Hemat., 12 (1972).

[20] Jensen, W. N. Rucknagel, D. L., Taylor, W. J., J.laborat. clin. Invest., 56, 854 (1960).

[21] Singer, K., Fisher, B., Perlstein, M. A., Blood, 7, 577 (1952).

[22] Reed, C., Ways, P., Simon, E., Nouv. Rev. fr. Hemat., 3, 59 (1963).

[23] Ling Miao, Bertrands Fourcade, Madan Rao, and Michael Wortis, Phys. Rev. A., 43, $6843(1991)$.

[24] J. C. Luke and J.I.Kaplan, Biophys. J., 25, 107 (1979).

[25] U.Serfeit and Lipowsky, Morphology of Vesicles, 420. In: Structure and Dynamics of Memberanes, Vol.1A, North-HOLLAND (1995).

[26] U.Serfeit, Advances in Physics 46, 35-37 (1997). 


\section{FIGURES}

FIG. 1. corniculate shape obtained from a destabilized sphere induced by osmotic pressure without constraints of constant area and volume. The corresponding location in the phase diagram is $\left(v=0.93, c_{0}=1.28\right)$.

FIG. 2. A quadri-concave shapes obtained by random searching under the constraint of constant volume. Its location in the phase diagram is $\left(v=0.84, c_{0}=-1.41\right)$.

FIG. 3. A sickle like shape obtained by random searching. The corresponding location in the phase diagram is $\left(v=0.74, c_{0}=-1.48\right)$.

FIG. 4. acanthocyte like shape obtained by random searching under the constraints of constant volume. The corresponding location in the phase diagram is $\left(v=0.56, c_{0}=1.89\right)$.

FIG. 5. The shape is obtained from Fig.4 by increasing the spontaneous curvature. All the buds in Fig.4 are evolved into "vesiculations", which shows a large positive $C_{0}$ favours the formation of "vesiculation". The corresponding location in the phase diagram is $\left(v=0.70, c_{0}=14.1\right)$.

FIG. 6. This is a perfectly sphere also obtained from Fig.4 by setting a zero spontaneous curvature, which shows a non-zero spontaneous curvature may be a condition for the formation of acanthocyte type shape. The corresponding location in the phase diagram is $\left(v=1.00, c_{0}=0\right)$.

FIG. 7. The pear-like shape is also evolved from Fig.4 under a negative spontaneous curvature, which shows a positive spontaneous curvature may be a necessary condition for the formation of the acanthocyte type shapes. The corresponding location in the phase diagram is $(v=0.89$, $\left.c_{0}=-3.25\right)$.

FIG. 8. A schematic copy of the phase diagram in SC model given by Seifert et al. in [5]. The location of the shapes found in the paper is denoted by $* n$ where $n$ corrsponds to Fig. $n$. In addition, Fig.5 $\left(v=0.70, c_{0}=14.1\right)$ is not denoted in it because the parameters are beyond the region of the phase diagram. 